\title{
Strategies for Radiology Reporting and Communication
}

\author{
Part 4: Quality Assurance and Education
}

\author{
Bruce I. Reiner
}

Published online: 4 December 2013

(C) Society for Imaging Informatics in Medicine 2013

\section{Introduction}

While the digitization of medical imaging practice has brought a number of dramatic changes in technology and workflow, perhaps the most understated change has been the change in physical layout and staff location, which has evolved from a central to distributed model [1]. In analog practice, the departmental layout and staff location were centrally and group focused, with both the radiologist reading room and technologist working area created to accommodate large groups of individuals, which routinely interacted with one another for the purposes of peer-topeer education, quality assurance, and case review. This naturally created an atmosphere of ongoing communication, collegiality, and mentoring. This trend of group dynamics and interaction extended to the medical staff, which by necessity had to physically travel to the radiology department to review imaging studies ordered on their patients. With the transition to filmless operation, this centralized working model was replaced by a distributed model, in which radiologists and technologists work in relative independence to one another. While this was intended to improve workflow and operational efficiency, the unintended impact has been diminished interpersonal communication, peer review, and education. As access to medical imaging data has become ubiquitous and instantaneous with the adoption of the picture archival and communication system, this distributed practice model has also extended into the referring physician community, resulting in decreased face-to-face communications between radiologists and referring physicians [2]. This distributed model of radiology

\section{B. I. Reiner $(\bowtie)$}

Department of Radiology,, Veterans Affairs Maryland Healthcare System, 10 North Greene Street, Baltimore MD 21201, USA

e-mail: breiner1@comcast.net practice has become further magnified with teleradiology, with the outsourcing of professional radiologist services to remote locations and independent (i.e., third party) service providers $[3,4]$.

Parallel changes outside of medicine have dramatically changed the manner and speed with which individuals communicate and interact with one another. Direct dialogue has been largely replaced by electronic communication, which can take place through text messaging, instant messaging, and e-mail. While this shift towards electronic communication has had a positive impact on communication workflow and timeliness, it has also led to a diminution in the degree of interpersonal connection between the communicating parties $[5,6]$. While computer-based face-to-face communication options do exist (e.g., Skype), they are relatively impractical in the setting of quick and concise communications which are routinely customary within everyday medical imaging practice. In these situations, it is less important to see one another's face than it is to see the imaging dataset, which is the central component of the communication [7]. The ideal solution is to, therefore, create a technology which fosters communication in a fast and intuitive manner, incorporates some degree of familiarity and individuality into the communication process, and directly integrates the imaging dataset into the communication platform.

\section{The Vital Role of Communication in Education and Quality Improvement}

Historically, the principal manner in which healthcare professionals learn and solve a problem is through peer-to-peer communication [8-10]. Traditional radiology quality assurance (QA) practice, which entails image quality analysis, has been performed in a collective fashion using the combined wisdom of multiple technologists and/or radiologists. 
Combining the collective experience and education of multiple parties serves as an excellent means to share knowledge while also adding some degree of impartiality to the process of image analysis. Unfortunately, the current model of QA practice often relies on a single assessment of image analysis which is performed by the same technologist tasked with image acquisition. This dual responsibility has the potential to introduce bias in the image quality analysis, along with a lack of shared knowledge and insight [11]. The ideal solution would be to create a methodology where QA can be performed in a collective and unbiased fashion, but without introducing workflow and time-intensive limitations. This collective QA analysis could have the additional theoretical benefit of improving technologist education and longitudinal job performance by providing direct QA feedback based upon the collective knowledge and experience of multiple unbiased stakeholders.

This dual benefit of using peer-to-peer communication to improve quality and education also extends to radiologists, which also currently operate in a distributed work model and are often isolated from their peers. This results in decreased peer-to-peer communication, which becomes further exacerbated by heightened productivity expectations. The opportunities for quality improvement in everyday radiologist practice can be seen in peer review, realtime consultations, and postreporting feedback. Enhanced communication lies at the center of these quality improvement opportunities, but is limited by existing workflow and technologies. A radiologist providing a consultation to a peer is currently required to disrupt existing workflow, open the case in question, review the imaging dataset (along with relevant historical imaging studies and reports) and then initiate communication through either a telephone conversation or back and forth electronic communications (e.g., instant messaging). The disruptive nature of the existing communication and negative impact on workflow and productivity ultimately discourages peer-to-peer consultations, which, in turn, limits educational opportunity and has the potential to negatively impact report diagnostic accuracy and confidence.

The lost opportunity for education and quality improvement through enhanced communication also extends into interactions between different stakeholder groups and individual steps in the medical imaging chain. In the steps of exam ordering, enhanced communication between the referring clinician and radiologist can result in quality improvement by optimizing exam selection as well as providing valuable clinical data for interpretation. In the step of protocol optimization, enhanced communication between the technologist and radiologist can theoretically lead to quality improvement through enhanced patient safety (e.g., radiation dose reduction), contrast optimization, and image quality. With improved communication comes the sharing
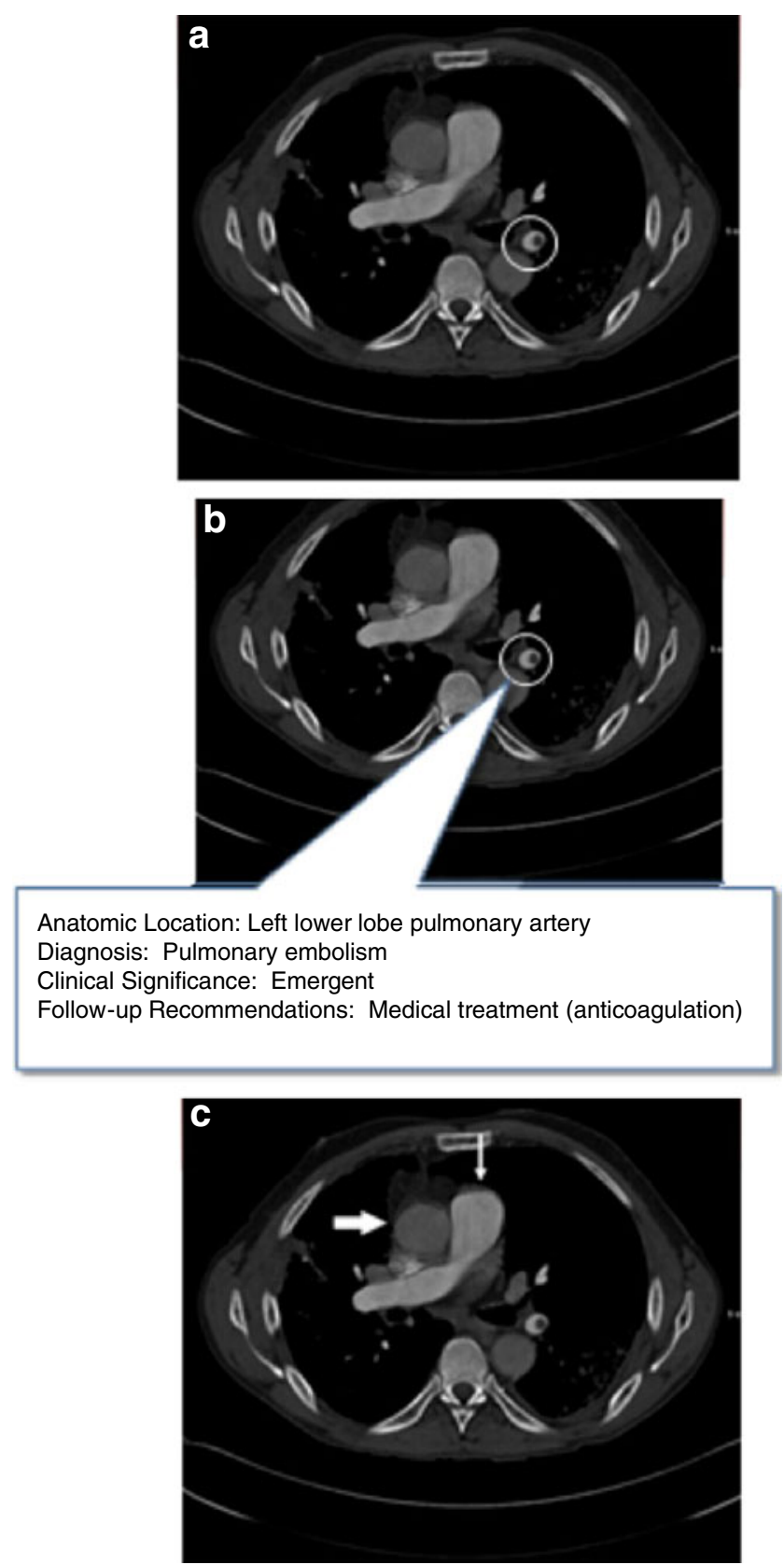

Fig. 1 Multifunctionality of key image annotation. The concept of annotating key images can be applied to a number of applications including (but not limited to) results communication, peer review, education, quality assurance (QA), and interactive consultation. a A single key image is selected from a chest CT angiogram showing a pulmonary embolus in the left lower lobe pulmonary artery (circled). b The corresponding data related to diagnosis, clinical significance, and follow-up recommendations are directly linked to the image annotation, and can be automatically viewed by highlighting the annotation. $\mathbf{c}$ The same key image can also be used for separate QA purposes, by annotating the specific region of interest and providing QA feedback. In this example, the ascending thoracic aorta (large arrow) and main pulmonary artery (thin arrow) are highlighted to show excellent bolus timing, resulting in selective opacification of the pulmonary artery relative to the thoracic aorta and a resulting exemplary QA score 
of knowledge and experience, along with the potential to reduce medical error and improve clinical outcomes. The ultimate goal is to facilitate communication at the point of care, where its clinical impact is of greatest value.

\section{Creating a Practical Solution}

The concept of creating a communication strategy utilizing a standardized annotation and mark-up schema for individually selected key images was described in an earlier article in this series [12]. The proposed workflow would consist of selecting a key image from the comprehensive imaging dataset, annotating the image using a standardized markup schema (along with an option for incorporating additional free text) and then electronically routing the annotated images to selected end users.

\section{a}

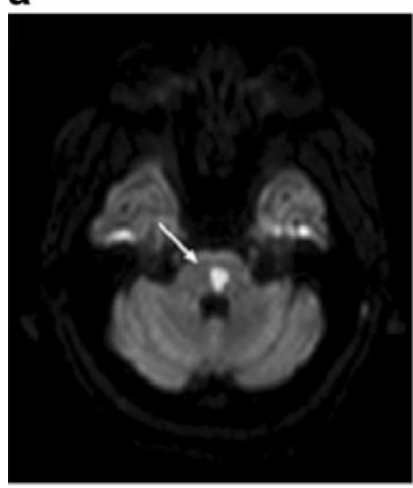

C

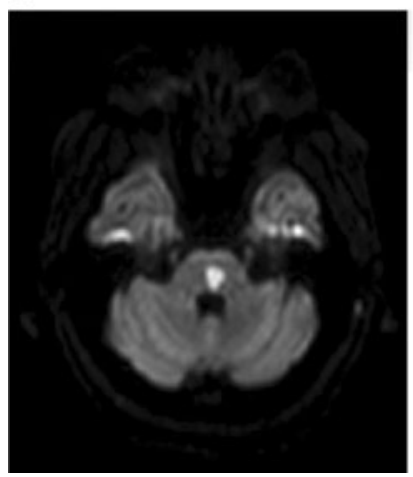

b
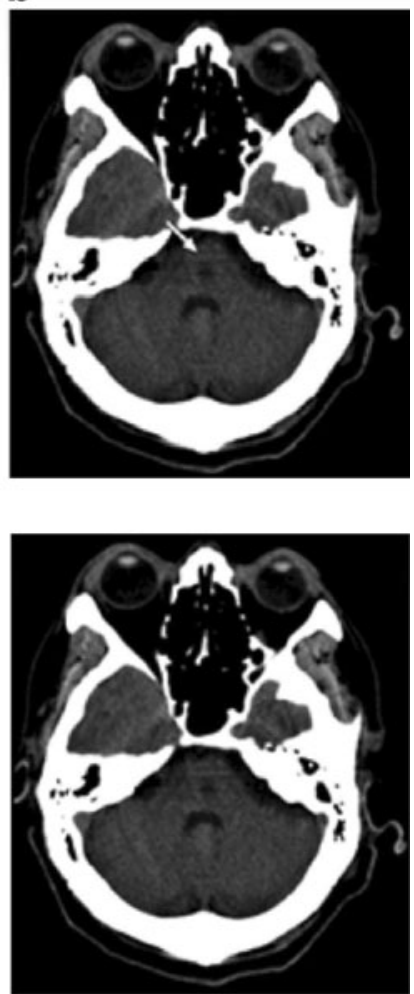

Fig. 2 Use of key image annotation for colleague feedback and peer review. a During the course of interpretation of a brain MRI for a suspected stroke, an acute brainstem infarct is identified (arrow) on a diffusion image. b Correlation with a CT performed $12 \mathrm{~h}$ earlier revealed the same finding (arrow), which was undiagnosed on the earlier report. $\mathbf{c}$ The selection of corresponding key images from the sequential CT and MRI exams can, in turn, be used to notify the radiologist who had interpreted the head CT of the discrepancy, along with generating a peer review report. The goal is to provide educational feedback, which is often lacking in conventional practice, while also creating an image-centric QA tool
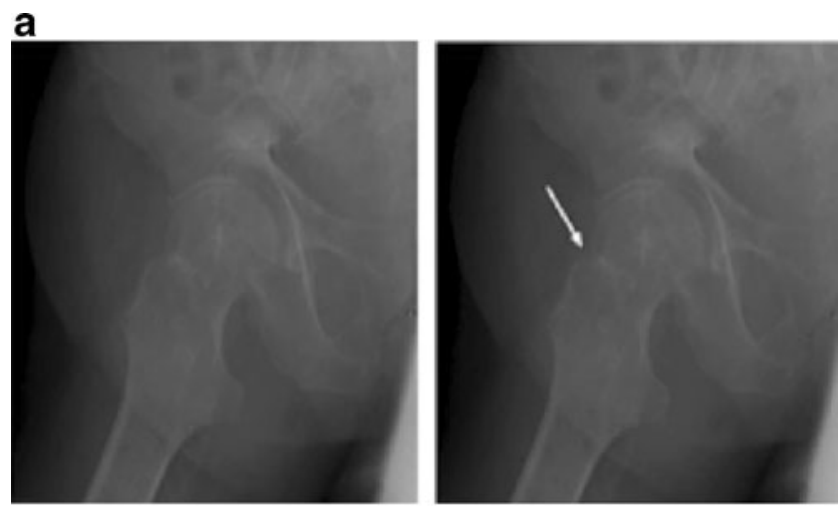

b
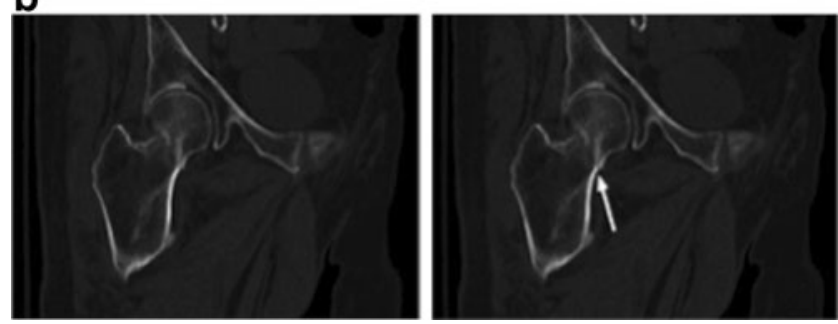

Fig. 3 Emergency room (ER) discrepancies and tracking of follow-up recommendations. In conventional practice, many imaging exams are initially reviewed by clinicians (e.g., ER and ICU) prior to radiologist interpretation. In the event that the clinician and radiologist interpretations are divergent, it is essential that truth be promptly established and acted upon in order to avoid adverse clinical outcomes. In the current model of image interpretation and clinical follow-up, deficiencies in communication have the potential to introduce error and/or delay in effective clinical diagnosis and management. One theoretical solution for improvement is to utilize a standardized mechanism for recording, annotating, and tracking key image data across the continuum of care. a A frog leg view of the right hip (with and without annotations) shows a subtle contour irregularity at the right femoral head-neck junction suspicious for (but not diagnostic of) a nondisplaced fracture, which was not identified by the ER physician. b In the interpretation and creation of the right hip radiograph report, the radiologist notified the ER of the possible discrepancy by annotating the key image and recommending a CT for definitive diagnosis. The resulting coronal CT images (with and without annotations) confirm the presence of an acute nondisplaced right hip fracture

Table 1 Data tracking of imaging QA discrepancy and peer review

\section{Exam type}

2. Date and time of exam

3. Date and time of interpretation

4. Interpreting radiologist or clinician

5. Type of QA discrepancy

6. Established diagnosis

7. Date and time discrepancy documented

8. Method of discrepancy detection

9. Individual reporting discrepancy

10. Notification confirmation to original interpreting physician

11. Impact on clinical care

12. Actions taken 
Fig. 4 Real-time interactive consultation. A portable chest radiograph performed in an intensive care unit (ICU) patient to evaluate endotracheal tube placement shows the endotracheal tube terminating at the level of the carina (arrow in left image), with an optimal position $4 \mathrm{~cm}$ proximal to the current position (annotated right image). a By selectively annotating the image, the radiologist can effectively communicate with the ICU physician in real time, with confirmation and acknowledgment performed electronically. b The follow-up image obtained after endotracheal tube repositioning shows satisfactory location of the endotracheal tube (long arrow) as well as the right internal jugular catheter (short arrow)

\section{a}
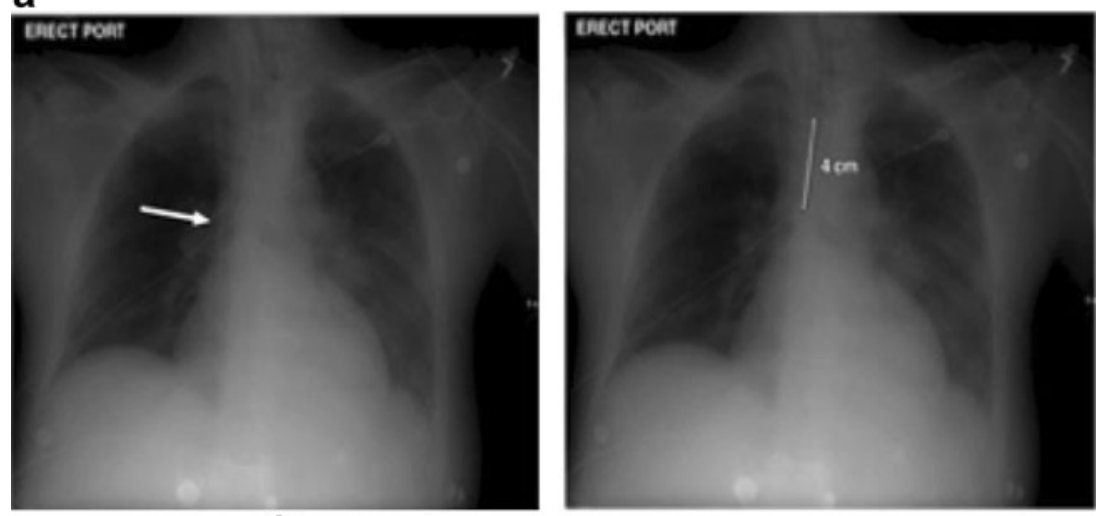

b

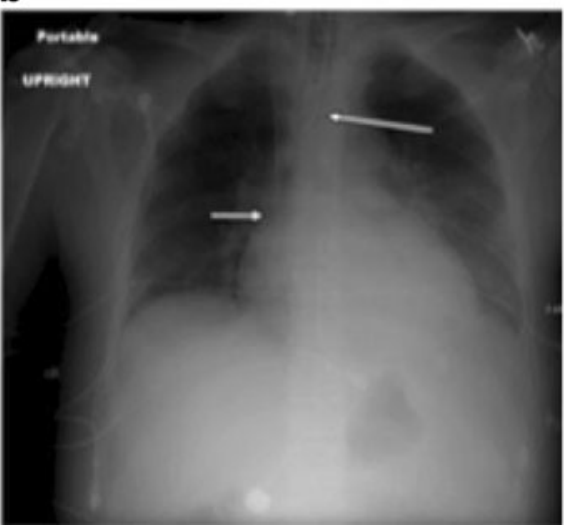

In order to illustrate how the proposed strategy would work, we will use the example of a radiologist tasked with interpretation of a chest CT angiogram for evaluation of pulmonary embolism. As the radiologist navigates through the comprehensive imaging dataset, he/she processes a variety of data related to clinical data, imaging protocol, image processing, image quality, pathologic findings, and diagnosis. In most situations, however, information related to many of these various data will not be formally communicated or acted upon. The radiologist output will customarily consist of a report which documents the imaging findings (both positive and negative) along with a diagnosis (or differential diagnosis). On occasion, the radiologist may want to communicate with the referring physician, performing technologist, or radiologist colleague. In order to do so, the radiologist will have to stop what he/she is doing, initiate contact with the individual of interest, and engage in a dialogue specific to the issue of concern. These steps are often performed in a discontinuous fashion, with intermittent delays. Once the communication has taken place, additional time delays take place if the other party is required to access and review the imaging data in question. The resulting time delays and interruptions in workflow not only have the unintended effect of diminishing productivity for both parties but also have the potential to introduce errors or oversights as individuals migrate between different tasks and imaging studies. Studies have shown that multitasking creates higher memory load, which can contribute to medical errors $[13,14]$.

A preferable solution would be to create a method for integrating the imaging data directly into the communication process by preselecting the individual images of interest (i.e., key images) and annotating the specific area/s within the images of interest. If the annotation schema used is standardized, two fundamental benefits can be derived. First, the repetitive use of a standardized annotation and mark-up schema will create familiarity and understanding on the part of the end users over time. Secondly, a standardized annotation schema can be used to create standardized graphical and textual data, which can, in turn, be populated into a referenceable database for future data mining and analytics. Upon completion of image annotation, the end user can initiate an automated communication sequence, which utilizes the individual communication preferences of each party, along with a prioritization schema defined by the significance and time requirements of the specific type of communication. Examples of different degrees of urgency and timeliness for communications related to the example of a chest CT angiogram could include critical results communication to a referring physician (emergent and immediate), consultation with a radiologist colleague (urgent with a short turnaround time required [e.g., within 10 minutes]), and image quality feedback to a technologist (nonemergent and no time restrictions). 
In each scenario, the reporting radiologist would annotate the key image, record the intended function (e.g., critical results reporting, interpretation consultation, and QA feedback), and designate the intended communicant. The resulting communication sequence would be electronically audited to ensure receipt and acknowledgment of the communication within a predefined period of time, in accordance with the institutional and individual end users' requirements. The specific method of communication can be customized to individual end user's preferences, with built-in automated escalation pathways to ensure communication success. In the example of an emergent/urgent communication, receipt and acknowledgment failure within a predefined period of time (e.g., $5 \mathrm{mi}-$ nutes) would, in turn, trigger the escalation pathway, so that the individual "next in line" would be emergently notified of a "failure to receive" emergent communication. All events within the communication pathway would be automatically recorded in a communication database, thereby providing an electronic audit for quality assurance.

This electronic communication database would record a number of referenceable data, which can be used for quality assurance, clinical management, education, research, technology development, and creation of best practice guidelines. Figure 1 demonstrates how the communication tool and recorded data can be used to communicate emergent imaging findings, consult with the referring physician, and ensure follow-up recommendations are performed in a timely fashion. In this example, appropriate follow-up would consist of initiation of thrombolytic or anticoagulation therapy, which would be documented in the physician medication orders. In the course of placing this order, the referring physician can provide a data link between the key image and pharmacy order (by referencing the pharmacy order tracking number and the key image finding), thereby integrating imaging and clinical data. An alternative computer-generated (i.e., automated) link can be established through the use of artificial intelligence techniques (e.g., neural networks) [15]. The net result is that when the key image is opened in future, the chain of communication and clinical management events would all be accessible to the reviewer, thereby providing a view of events in the continuum of care.

In a similar manner, the communication tool can also be used by interpreting radiologists to provide important feedback relating to prior interpretation or report discrepancies (Figs. 2 and 3). This can take a number of forms including a finding described on a prior radiologic report (e.g., radiologist peer review), finding from a clinician interpretation of the same radiologic exam (e.g., ER QA discrepancy), or finding on a preliminary resident-generated report (e.g., resident education). In all examples, the annotated key images and supporting text data would provide a simple, concise, and straightforward method for alerting colleagues of discrepancies in interpretation, along with the ability to interactively communicate with one another using the annotated key image as the principle data source. A concurrent database can be created to track associated data related to the date/time, participants, communication, and follow-up actions taken (Table 1).

While these communication strategies offer the potential for improved education and peer review, they are largely retrospective in nature, which limits the intended goal of real-time intervention. One application of the proposed tool which could provide real-time intervention at the point of care is communication in the critical care patient. In Fig. 4, a commonly experienced sequence of events is illustrated in which a critical care patient has undergone placement of a central venous catheter and endotracheal tube. By annotating the key image, the radiologist can communicate to the intensive care physician or nurse the positioning of these lines, along with recommendations for adjustment. Upon receipt of the annotated key image, the clinical care provider can confirm receipt; acknowledge the abnormality in question; consult with the radiologist, if needed; and order a repeat imaging exam following intervention.

The intended goal of the proposed communication tool is to directly integrate imaging data into communication in the multitude of steps and stakeholders of the medical imaging chain, without causing interruptions and unnecessary delays in workflow. By standardizing the data used, referenceable databases can be derived offering a number of deliverables including education, research, quality improvement, data mining, and integration of imaging and clinical data.

\section{References}

1. Reiner B (2013): Creating accountability in image quality analysis. Part I: the technology paradox. J Digit Imaging (in press).

2. Reiner B, Siegel E, Protopapas Z, et al: Impact of filmless radiology on the frequency of clinician consultations with radiologists. AJR 173:1169-1172, 1999

3. Thrall JH: Teleradiology. Part I: history and clinical applications. Radiology 243:613-617, 2007

4. Wachter R: The "dis-location" of U.S. medicine: the implications for medical outsourcing. NEJM 354:661-665, 2006

5. Papacharissu Z, Rubin A: Predictors of Internet use. J Broadcast Electron Media 44:175-195, 2000

6. Markus ML: Finding a happy medium: explaining the negative effects of electronic communication on social life at work. ACM Trans Inf Syst (TOIS) 12:119-149, 1994

7. Reiner BI, Siegel EL: Radiology reporting: returning to our imagecentric roots. AJR 187:1151-1155, 2006

8. Evans DJR, Cuffe T: Near-peer teaching in anatomy: an approach to deeper learning. Anat Sci Educ 2:227-233, 2009

9. Allen AR, Boraks N: Peer tutoring: putting it to the test. Read Teach 32:274-278, 1978

10. Gill D, Parker C, Spooner M, et al: Tomorrow's doctors and nurses: peer assisted learning. Clin Teach 3:13-18, 2006 
11. Reiner BI: Automating quality assurance for digital radiography. J Am Coll Radiol 7:486-490, 2009

12. Reiner B: Innovation strategies for radiology reporting and communication. Part II: physician communication. (in press)

13. Taylor GA, Voss SD, Melvin PR, Graham DA: Diagnostic errors in pediatric radiology. Pediatr Radiol 41:327-334, 2011
14. Laxmisan A, Hakimzada F, Sayan OR, et al: The multitasking clinician: decision-making and cognitive demand during and after team handoffs in emergency care. Int J Med Inf 76:801-811, 2007

15. Stead WW, Miller RA, Musen MA, Hersh WR: Linking information from disparate sources and into workflow. J Am Med Inform Assoc $7: 135-145,2000$ 